\title{
Intrafollicular expression of matrix metalloproteinases and their inhibitors in normally ovulating women compared with patients undergoing in vitro fertilization treatment
}

\author{
Sandra D’Ascenzo ${ }^{1}$, Ilaria Giusti ${ }^{1}$, Danilo Millimaggi ${ }^{1}$, Roberto Marci $^{2}$, Carla Tatone ${ }^{2}$, Rosella Cardigno Colonna ${ }^{2}$, \\ Massimo Moscarini ${ }^{3}$, Antonio Pavan ${ }^{1}$, Vincenza Dolo ${ }^{1}$ and Donatella Caserta ${ }^{1,2}$ \\ ${ }^{1}$ Department of Experimental Medicine and ${ }^{2}$ Fertility and Sterility Unit, University of L'Aquila, L'Aquila, Italy and ${ }^{3}$ Sant'Andrea Hospital La Sapienza, \\ University of Rome, Rome, Italy \\ (Correspondence should be addressed to D Caserta, Department of Experimental Medicine, Via Vetoio-Coppito, L'Aquila 67100, Italy; Email: \\ donatellacaserta@virgilio.it)
}

\begin{abstract}
Objective: To assess possible differences in the activity of matrix metalloproteinases (MMPs), MMP-2 and MMP-9, and their inhibitors, the tissue inhibitors of MMPs, TIMP-1 and TIMP-2, in follicular fluid (FF) of women undergoing in vitro fertilization (IVF) treatment and of normally ovulating women.

Design: Prospective study.

Methods: MMP-2 and MMP-9 activity was analyzed by gelatin zymography and MMP-2, MMP-9, TIMP-2, TIMP-1 and $17 \beta$-estradiol levels were measured in FF by ELISA.

Results: We found significantly reduced MMP levels in FF of women undergoing IVF treatment when compared with those of normally ovulating women. In contrast, the TIMP-1 levels were found significantly increased in FF from IVF patients vs normally ovulating women. No significant differences were found for TIMP-2 between the two groups.

Conclusions: These findings underline a marked difference in MMPs and their inhibitors in the IVF women and the control group. Therefore we assume MMPs depend on hormonal steroidogenesis modulation induced by the gonadotropin protocol for IVF treatment.
\end{abstract}

European Journal of Endocrinology 151 87-91

\section{Introduction}

Matrix metalloproteinases (MMPs) and tissue inhibitors of metalloproteinases (TIMPs) play very important roles in extracellular matrix (ECM) remodeling in many physiological conditions, including ovarian follicular growth and ovulation (1-2). Specific aspects of follicular development, ovulation and subsequent formation and regression of the corpus luteum are dynamic events, largely performed by MMPs (3). MMPs and TIMPs are controlled by hormones, growth factors and cytokines, and are involved in critical changes in the structure and function of reproductive organs (4).

Although the role of extracellular MMPs in ovarian tissue remodeling during the life span of the follicle has been demonstrated, very few data report direct evidence for a relationship between human oocyte aging and the induction of matrix-degrading proteases and their inhibitors $(5-7)$.

The balance between the level of active MMPs and available TIMPs determines the net MMP activity and therefore has a pivotal role in ECM turnover. This balance among MMPs and TIMPs is thought to be important in various pathologies including arthritis, cancer and tumor invasion as well as in physiological processes, such as follicular development and rupture (8). Follicular development and the rupture of the follicular wall that takes place at the time of ovulation are gonadotropin-induced processes that involve extensive tissue remodeling. Accordingly, proteolytic enzymes such as plasminogen activators and MMPs are expressed in the ovary in response to gonadotropins. Indirect studies suggest that these proteinases provide a cascade of proteolytic activity required for follicular development and rupture of the follicular wall $(9,10)$.

The mechanisms controlling the production and activation of MMPs in vivo under physiological conditions are not well understood.

In this study, we present for the first time a comparative study of MMP and TIMP levels detected in follicular fluid (FF) of normally ovulating women and of patients 
undergoing in vitro fertilization (IVF) treatment, relating them to $17 \beta$-estradiol levels.

\section{Materials and methods}

\section{Patients and induction of ovulation}

The study population included 11 women with natural cycles and 11 women under IVF treatment selected for male factor infertility.

The FF was collected during oocyte pick-up from women under IVF treatment (IVF Group). At the same time FF was collected during gynecological surgery from 11 pluriparous women (Control Group) with no stimulation treatment whatsoever. The FF single aspiration in the Control Group was performed during laparotomy procedure using a 30-gauge needle connected to a $5 \mathrm{ml}$ syringe.

All FFs from the IVF Group and the Control Group used in this research come from a single-follicle aspiration. Exclusion criteria for this group were: infertility problems, endometriosis, ovarian cyst and genital cancer.

All ovarian stimulation in the IVF Group was performed by using a long down-regulation protocol with a gonadotropin-releasing hormone agonist (Enantone 3.75; Takeda) on the 23rd day. Therapy was continued daily with 225 follicle-stimulating hormone r IU (Gonal F; Serono) from day 3 of the subsequent menstruation with an estradiol level $<40 \mathrm{pg} / \mathrm{ml}$.

Every 2 or 3 days, follicular monitoring was performed by serum estradiol measurement and serial transvaginal ultrasound. When three or more follicles $>18 \mathrm{~mm}$ in diameter were seen on transvaginal ultrasonography, patients received 10000 IU human chorionic gonadotropin (hCG) (Profasi; Serono). Transvaginal ultrasonography-guided follicular aspiration (pick-up) was scheduled $35-36 \mathrm{~h}$ after hCG administration. In the Control Group, the women underwent surgery after transvaginal ultrasound examination. They were all in follicular phase, with follicles ranging from 18 to $20 \mathrm{~mm}$ in diameter. All the patients in both groups were enrolled after signing a written consent to participate in the study.

After oocyte removal, FF samples were centrifuged at $800 \boldsymbol{g}$ for $10 \mathrm{~min}$, and supernatants were immediately processed. Blood-free, yellow-colored, undiluted FF samples were obtained from each patient. The protein content of each sample was determined using the method of Bradford (Bio-Rad; Milan, Italy) with BSA (Sigma) as the standard.

\section{Zymographic analyses}

Proteolytic activity of MMPs was determined in an equal volume of one aspirated clear FF from the two groups of women using gelatin zymography.
Zymography was performed using $7.5 \%$ SDS-PAGE gels co-polymerized with $1 \mathrm{mg} / \mathrm{ml}$ gelatin type $B$ (Sigma). All FFs were diluted 1:10 with PBS and mixed with SDS-PAGE sample buffer in non-reducing conditions without heating. After electrophoresis, gels were washed twice for $30 \mathrm{~min}$ in $2.5 \%$ Triton X-100 at room temperature and incubated overnight in collagenase buffer $(50 \mathrm{mM}$ Tris-HCI, pH 7.5, $10 \mathrm{mM}$ $\left.\mathrm{CaCl}_{2}, 150 \mathrm{mM} \mathrm{NaCl}\right)$ at $37^{\circ} \mathrm{C}$. Gels were stained in Coomassie Blue R 250 (Bio-Rad) in a mixture of methanol:acetic acid:water (4:1:5) for $1 \mathrm{~h}$ and unstained in the same solution without dye.

Gelatinase activities were visualized as white bands, indicating proteolysis of the substrate. Every sample was assayed in duplicate and every experiment was performed at least twice. The same results were also observed when the MMP activity was evaluated, loading a known weight of the proteins $(\mu \mathrm{g})$.

\section{ELISAs for MMPs, TIMPs and $17 \beta$-estradiol}

The concentrations of MMP-2 and MMP-9 in human FFs were determined in duplicate by using commercial kits, the Quantikine MMP-2 and Quantikine MMP-9 ELISA Kit (R\&D System, Minneapolis, MN, USA). The assays employed the quantitative sandwich enzyme immunoassay technique designed to measure total MMP-2 (pro- and active MMP-2) and total (pro- and active form) of MMP-9. The assay sensitivity for MMP-2 was $0.03 \mathrm{ng} / \mathrm{ml}$ and $0.156 \mathrm{ng} / \mathrm{ml}$ for MMP-9.

TIMP-1, TIMP-2 and 17ß-estradiol ELISA Kits were purchased from Oncogene (Oncogene Research Products, San Diego, CA, USA). The assay sensitivity for TIMP-1 was $0.0096 \mathrm{ng} / \mathrm{ml}, 3 \mathrm{ng} / \mathrm{ml}$ for TIMP-2 and $28 \mathrm{pg} / \mathrm{ml}$ for $17 \beta$-estradiol.

\section{Statistical analysis}

Statistical analysis of the data was performed using Student's t-test when two groups were compared. Data are presented as means \pm S.D. In all cases, $P<0.05$ was considered statistically significant.

\section{Results}

\section{Patient characteristics}

In this study, we analyzed 11 women without fertility problems (Control Group) and 11 women undergoing IVF treatment selected for male factor infertility (IVF Group). The average age of the normally ovulating women was around 40, and 37 for the IVF patients. All FFs were collected from follicles which had reached a mean diameter ranging from 18 to $20 \mathrm{~mm}$. The collected FF volume was similar at $\sim 3.5 \mathrm{ml}$ in both clinical groups. All these patient parameters are reported in Table 1. 
Table 1 Characteristics of study patients: values are means \pm S.D.

\begin{tabular}{lcc}
\hline Characteristics & $\begin{array}{c}\text { Normal patients } \\
(n=11)\end{array}$ & $\begin{array}{c}\text { IVF patients } \\
(n=11)\end{array}$ \\
\hline Age (years) & $40 \pm 7.6$ & $37 \pm 5.2$ \\
Follicular fluid volume $(\mathrm{ml})$ & $\sim 3.5$ & $\sim 3.5$ \\
Follicular diameter $(\mathrm{mm})$ & $18-20$ & $18-20$ \\
\hline
\end{tabular}

\section{MMP-2, MMP-9, TIMP-1, TIMP-2 and $17 \beta$-estradiol levels in FF}

The presence of MMPs in each sample of aspirated FF was analyzed by gelatin zymography and ELISA. Lytic bands corresponding to MMP-2 and MMP-9 were observed (Fig. 1). The Control Group showed higher MMP-2 levels compared with the IVF. A similar trend was seen for MMP-9; in many samples from the IVF Group this enzyme was not detected. These gel observations were confirmed by quantitative determinations using an ELISA immunoassays (Fig. 2A and B). There is some variability within the Control Group results, probably due to the unique physiology of each woman, whereas all the MMP-2 levels of the IVF Group seem to be similar (Fig. 2A). The value, reported as $\mathrm{ng} / \mathrm{ml}$ of total MMP-2 (active and pro-enzyme form), shows a significant difference in MMP-2 levels between the Control Group and the IVF Group $(P<0.002)$. The presence of MMP-9, reported as $\mathrm{ng} / \mathrm{ml}$ of total MMP9, was not detected in IVF patients, while the Control Group high MMP-9 levels observed were quite high (Fig. 2B). Using a quantitative ELISA method, we also checked the levels of TIMP-2 and TIMP-1 (Fig. 2C and D). Both inhibitors were clearly detectable in all FFs; no significant differences were found for TIMP-2

\section{IVF}

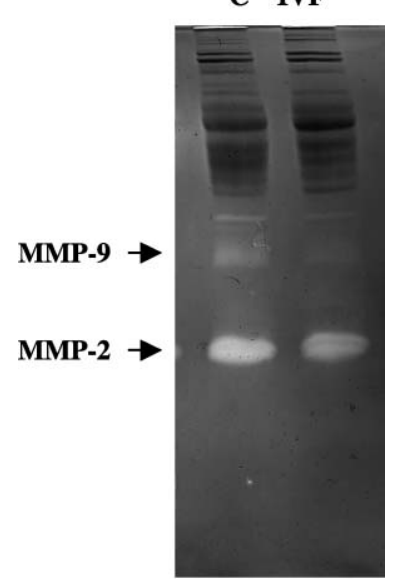

Figure $1 \mathrm{~A}$ representative zymography of FF from women without fertility problems (C) and women undergoing IVF treatment selected for male factor infertility (IVF). Samples were processed as described in Materials and methods. Two lytic bands of gelatinolytic activity, corresponding to MMP-2 and MMP-9, were detected in both groups: the control showed higher levels compared with IVF patients. between IVF patients and normally ovulating women (Fig. 2C). On the other hand, a great difference was visible for TIMP-1 between the two groups; the level of TIMP-1 seems to have an even rate inside the IVF Group (Fig. 2D).

As reported in the literature, ovarian function is based mainly on measuring serum estradiol levels, but $17 \beta$ estradiol serum level is produced from all kinds of follicles present in the ovaries during the IVF treatment. In assisted reproductive technologies cycle the goal is to retrieve mature oocytes and the parameter of maturity is closely associated with follicular size.

Our aim was to evaluate a possible correlation in a single FF between MMPs and 17ß-estradiol in treated and non-treated patients. We measured the amount of $17 \beta$-estradiol in the FFs and sera of every patient.

The data reported in Fig. 3 show a significant difference in FF 17ß-estradiol levels between the groups $(P<0.05)$. In particular, the FF $17 \beta$-estradiol value in IVF women (mean of 11 patients $=654 \mathrm{ng} / \mathrm{ml}$ ) is 2.2-fold lower than in normally ovulating women (mean of 10 patients $=1477 \mathrm{ng} / \mathrm{ml}$ ). In contrast we found that serum $17 \beta$-estradiol levels from IVF patients $(1000-6700 \mathrm{pg} / \mathrm{ml})$ were remarkably higher with respect to the values of the normally ovulating women $(48-427 \mathrm{pg} / \mathrm{ml})$. We can explain this if we consider that $17 \beta$-estradiol serum of IVF patients is due to follicles of all different diameters present in the ovary, while in the normally ovulating women the $17 \beta$-estradiol serum value comes from a single follicle.

\section{Discussion}

In this study, we demonstrate, for the first time, that MMP-2 and MMP-9 expression was much lower in FFs of IVF patients compared with what was found in normally ovulating women. In addition, we found similar TIMP-2 levels in both clinical groups, while TIMP-1 levels were significantly higher in the IVF Group compared with controls.

Follicular development, ovulation, formation of corpus luteum and its regression involve extensive tissue remodeling. The development of ovarian follicles and the breakdown of the follicular wall to release the mature oocyte at the time of ovulation, requires extensive angiogenesis and remodeling (9); the resulting mature follicle remains in an extracellular environment of collagen, laminin and fibronectin. Researchers have proposed that the extensive changes in the ovarian ECM during folliculogenesis are accomplished partially by the MMP system. Although a definitive causative effect has yet to be proved, correlative data implicate the MMP system in follicular growth. For example, Bagavandoss (11) observed that the pattern of MMP2 and MMP-9 immunolocalization was markedly increased at the later stage of follicular development. In other species, such as goats, collagenase activity 
A

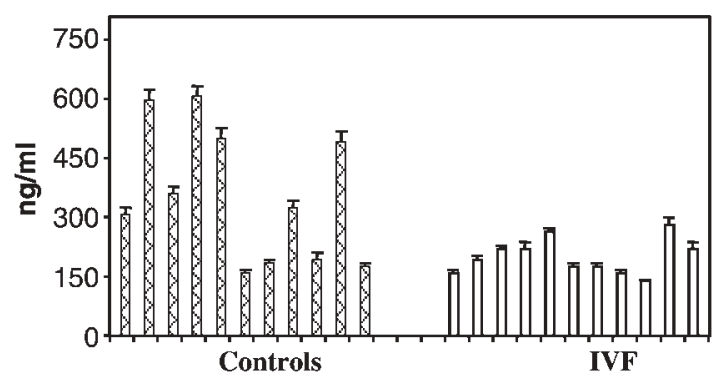

C

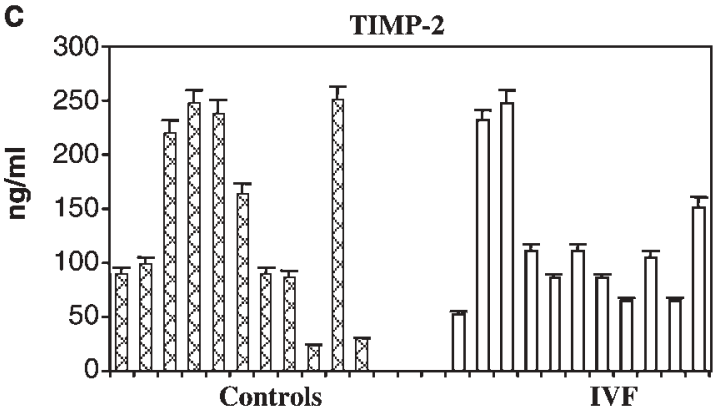

B

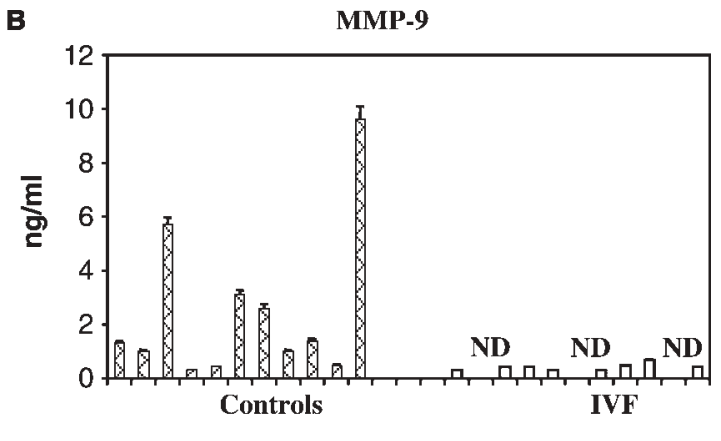

D

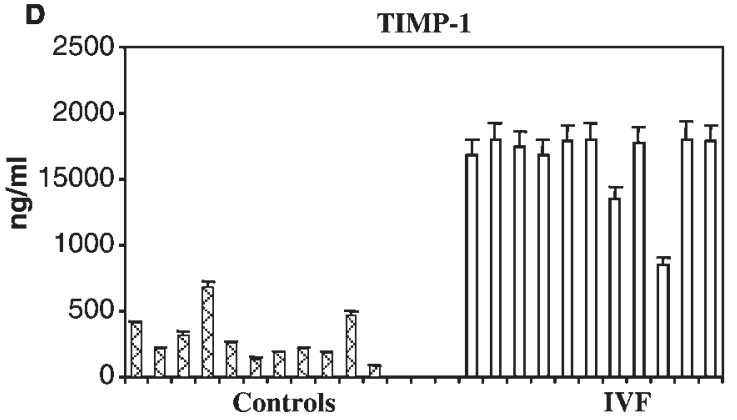

Figure 2 ELISA. Concentrations of MMP-2, MMP-9, TIMP-2 and TIMP-1 were detected in FFs from women without fertility problems (Controls) and women undergoing IVF treatment (IVF). Data are presented as means \pm S.D. from three independent experiments. ND, not detected.

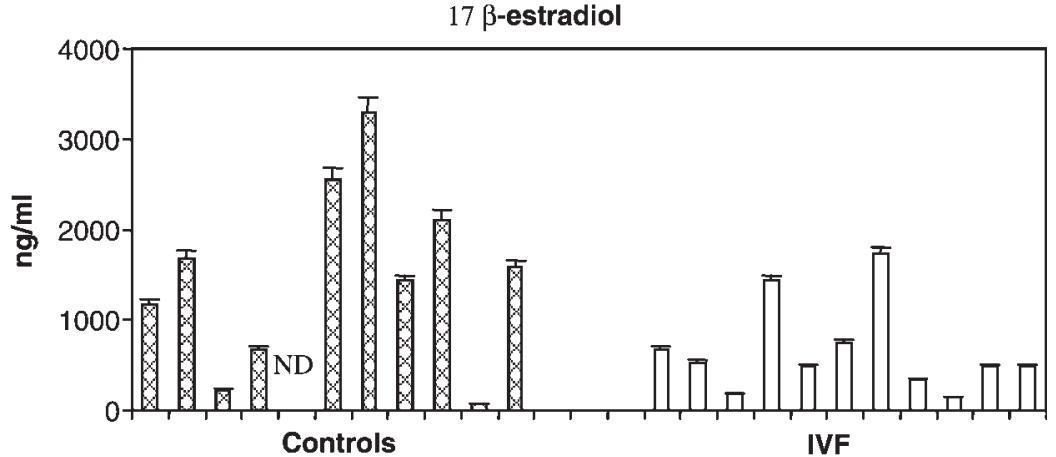

Figure 3 ELISA. Concentrations of $17 \beta$-estradiol were detected in FFs from women without fertility problems (Controls) and women undergoing IVF treatment (IVF). Data are presented as means \pm S.D. from three independent experiments. ND, not detected.

has been noted to increase with increasing follicular size (12). Although little is known about the MMPs during follicular growth in humans, in other species the MMPs are present within the follicle. Our results point out remarkable differences in the expression of MMPs and TIMPs in the two clinical groups enrolled in our two schedules. It seems that the presence of MMP-2 is higher (2-fold) in controls than in the IVF Group. The same behavior is more marked for MMP-9 level; as a matter of fact, in some cases we are not even able to detect the molecule in FF. We presume that MMP-2 is involve in follicular development, while MMP-9 expression is recruited in follicular breakdown.
The changes in the TIMPs during early follicular growth have received limited attention, but the emerging evidence suggests that the alterations in TIMPs are closely related to the changes in MMPs (13). A working hypothesis for the ovarian MMP system would encompass the fine balance of MMPs and inhibitors to regulate their action and localization. In this report we evaluated TIMP levels in the FFs of a normally ovulating control group vs those in IVF patients. TIMP-2 expression was similar in both clinical groups. However, we noticed a significantly higher TIMP-1 level in stimulated women. Expression of TIMPs is modulated by progesterone and estradiol and has been associated 
with steroidogenesis in granulosa cells (14). Examined together, our results lead us to hypothesize that the hormonal protocol in IVF patients induced a modification in the MMPs/TIMPs system during multiple follicular development. The MMP system may regulate normal follicular maturation and atresia to achieve the appropriate number of ovulatory follicles (12). Ovarian TIMPs may act as multifunctional autocrine/paracrine factors in cellular proliferation, differentiation, neovascularization or steroidogenesis during folliculogenesis.

The production of estradiol in the human ovary results from a cooperation of teca and granulosa cells (15); in clinical practice, serum estradiol is an established variable to detect multiple follicular development.

Although serum estradiol has been used to monitor the follicular growth during controlled ovarian stimulation, estradiol levels in FF may not play any role in predicting the quality of oocytes and embryos (16). In this study, our results failed to show any relationship between MMPs/TIMPs and FF estradiol levels.

This might suggest that some other variable is dominating the actual fertilizability of the oocytes, but that oocytes made in a follicle with well-functioning granulosa cells, once fertilized, are better fitted to start embryonic development.

In conclusion, further studies are needed to understand how the changes in the ratio of MMPs to TIMPs may reflect the ovarian response (follicular development and oocytes/embryo quality) during controlled ovulation stimulation.

\section{Acknowledgements}

This work was supported by grants from the Italian Ministry of University and Scientific and Technological Research (MIUR) and FIRB.

\section{References}

1 Matrisian LM. The matrix-degrading metalloproteinases. Bioessays $199214455-463$.

2 Birkedal-Hansen H. Role of matrix metalloproteinases in human periodontal diseases. Journal of Periodontology 199364 474-484.

3 Liu K, Olofsson JI, Wahlberg P \& Ny T. Distinct expression of gelatinase A [matrix metalloproteinase (MMP)-2], collagenase-3
(MMP-13), membrane type MMP 1 (MMP-14), and tissue inhibitor of MMPs type 1 mediated by physiological signals during formation and regression of the rat corpus luteum. Endocrinology $19991405330-5338$.

4 Hulboy DL, Rudolph LA \& Matrisian LM. Matrix metalloproteinases as mediators of reproductive function. Molecular Human Reproduction $1997327-45$.

5 Curry TE Jr, Sanders SL, Pedigo NG, Estes RS, Wilson EA \& Vernon MW. Identification and characterization of metalloproteinase inhibitor activity in human ovarian follicular fluid. Endocrinology $19981231611-1618$.

6 Curry TE Jr, Mann JS, Estes RS \& Jones PB. Alpha 2-macroglobulin and tissue inhibitor of metalloproteinases collagenase inhibitors in human preovulatory ovaries. Endocrinology 1990127 63-68.

7 Cossins J, Dudgeon TJ, Catlin G, Gearing AJ \& Clements JM. Identification of MMP-18, a putative novel human matrix metalloproteinase. Biochemical and Biophysical Research Communications $1996228494-498$.

8 Curry TE Jr \& Osteen KG. The matrix metalloproteinase system changes, regulation and impact throughout the ovarian and uterine reproductive cycle. Endocrine Reviews 200324 428-465.

9 Tsafriri A. Ovulation as a tissue remodelling process - proteolysis and cumulus expansion. Advances in Experimental Medicine and Biology 1995377 121-140.

10 Ny T, Peng XR \& Ohlsson M. Hormonal regulation of the fibrinolytic components in the ovary. Thrombosis Research $1993 \mathbf{7 1}$ $1-45$.

11 Bagavandoss P. Differential distribution of gelatinases and tissue inhibitor of metalloproteinase-1 in the rat ovary. Journal of Endocrinology $1998 \mathbf{1 5 8} 221-228$.

12 Garcia R, Ballesteros LM, Hernandez-Perez O, Rosales AM, Espinosa R, Soto H, Diaz de Leon L \& Rosado A. Metalloproteinase activity during growth, maturation and atresia in the ovarian follicles of the goat. Animal Reproduction Science $199747211-228$.

13 Grabiec M, Szymanski W, Szymanski M, Jendryczka J, Polak G, Gogacz M \& Rechberger T. Concentrations of MMP-1, TIMP-1, MMP-1/TIMP-1 and CTP complexes in follicular fluid as related to fertilization rate in women treated with in vitro fertilization. Ginekologia Polska 200172 107-112.

14 Ben-Shlomo I, Goldman S \& Shalev E. Regulation of matrix metalloproteinase-9 (MMP-9), tissue inhibitor of MMP, and progesterone secretion in luteinized granulosa cells from normally ovulating women with polycystic ovary disease. Fertility and Sterility $2003 \mathbf{7 9} 694-701$.

15 Erickson GF. Physiologic basis of ovulation induction. Seminars in Reproductive Endocrinology $1996 \mathbf{1 4} 287-297$.

16 Chang CL, Wang TH, Horng SG, Wu HM, Wang HS \& Soong YK. The concentration of inhibin $\mathrm{B}$ in follicular fluid in relation to oocyte maturation and embryo development. Human Reproduction $2002171724-1728$.

Received 16 December 2003

Accepted 26 February 2004 